\title{
Ganho de Peso e Características da Carcaça de Cordeiros Terminados em Pastagem Natural Suplementada, Pastagem Cultivada de Azevém (Lolium multiflorum Lam.) e Confinamento ${ }^{1}$
}

\author{
Cleber José Tonetto ${ }^{2}$, Cleber Cassol Pires ${ }^{3}$, Liziany Müller ${ }^{4}$, Marta Gomes da Rocha ${ }^{5}$, \\ José Henrique Souza da Silva ${ }^{5}$, Adriano Ramos Cardoso ${ }^{6}$, Diego Peres Neto 6
}

\begin{abstract}
RESUMO - Objetivou-se com este trabalho avaliar o desempenho e as características da carcaça de cordeiros, em três sistemas de alimentação. Foram utilizados 16 cordeiros, distribuídos aleatoriamente com suas mães, 24 horas após o nascimento, em três tratamentos: PNS: pastagem natural suplementada; PC: pastagem cultivada de azevém (Lolium multiflorum Lam.); e CON: confinamento. O ganho médio diário de $0,404 \mathrm{~kg}$ dos cordeiros da PC foi superior aos demais tratamentos, enquanto o ganho de $0,325 \mathrm{~kg} / \mathrm{dia}$ dos animais da PNS foi superior aos ganhos de $0,213 \mathrm{~kg} /$ dia dos animais do CON. O abate ocorreu quando os cordeiros atingiram em torno de $31 \mathrm{~kg}$. O peso da carcaça quente dos animais da PNS $(15,7 \mathrm{~kg})$ foi semelhante ao dos animais da PC $(16,8 \mathrm{~kg})$ e superior ao confinamento (14,3 kg). Os animais da PNS, PC e CON apresentaram resultados semelhantes para o índice de quebra ao resfriamento com valores de 2,3, 2,1 e $2,5 \%$, respectivamente. Os rendimentos da carcaça quente dos animais de PNS (50,2\%) e PC (53,7\%) foram semelhantes e superiores aos rendimentos dos animais do CON (45,9\%). A compacidade das carcaças não mostrou diferença entre PNS e PC, com valores de 0,287 e 0,307 , respectivamente, os quais, porém, foram superiores aos animais do CON $(0,253)$. As carcaças dos animais da PNS e CON não diferiram quanto à espessura de gordura com valores de 1,6 e 1,0 mm, respectivamente. Estes valores foram inferiores aos obtidos na PC (3,3 mm). Ganhos médios diários acima de $0,400 \mathrm{~kg} /$ dia e rendimento de carcaça quente acima de $52 \%$ podem ser obtidos com cordeiros alimentados com suas mães em pastagem cultivada de azevém, com peso de abate em torno dos $31 \mathrm{~kg}$.
\end{abstract}

Palavras-chave: carcaça quente, compacidade, espessura de gordura, ganho médio diário, sistemas de alimentação

\section{Weight Gain and Carcass Characteristics of Lambs Finished in a Natural Supplemented Pasture, Ryegrass Pasture (Lolium multiflorum Lam.) and Feedlot}

\begin{abstract}
The objective of this study was to evaluate the performance and carcass characteristics of lambs under three feeding systems. Sixteen lambs, 24 hours after birth, and their mothers were randomly assigned to three treatments: NSP: natural supplemented pasture; RP: ryegrass pasture (Lolium multiflorum lam.) and CON: confinement. Average daily gain of $0.404 \mathrm{~kg}$ of RP lambs was higher than the other treatments, whereas NSP animals showed higher gain $(0.325 \mathrm{~kg} /$ day $)$ than CON animals $(0.213 \mathrm{~kg} / \mathrm{day})$. The lambs were slaughtered when reached, approximately, $31 \mathrm{~kg}$. The carcass hot weight of NSP animals $(15.7 \mathrm{~kg})$ was similar to the RP animals $(16.8 \mathrm{~kg})$ and higher than the confinement ones $(14.3 \mathrm{~kg})$. NSP, RP and CON animals showed similar results to the cooling index, with values of $2.3,2.1$ and $2.5 \%$, respectively. Hot carcass yield of NSP (50.2\%) and RP (53.7\%) animals were similar, however those values were higher than those of CON carcass yield (45.9). Carcass compactness showed no difference between NSP and RP, with values of 0.287 and 0.307 , respectively, however, these values were higher than the CON animals $(0.253)$. The carcass of NSP and CON animals did not differ in relation to the fat thickness, with values of 1.6 and $1.0 \mathrm{~mm}$, respectively. These values were lower than the ones obtained from RP $(3.3 \mathrm{~mm})$. Average daily gain over $0.4 \mathrm{~kg}$ /day and hot carcass yield over $52 \%$ can be obtained in lambs fed with their mothers in ryegrass pasture, with slaughter weight round $31 \mathrm{~kg}$.
\end{abstract}

Key Words: average daily gain, compactness, fat thickness, feeding systems, hot carcass yield

\section{Introdução}

A introdução das raças de carne no sistema de produção ovina, no Rio Grande do Sul, apresenta-se como uma alternativa importante, sendo o cordeiro a categoria animal que possui alta velocidade de cres- cimento e rendimento de carcaça. Macedo \& Reis (1987) descrevem que a utilização de pastagens cultivadas de ciclo de inverno-primavera tem sido uma das alternativas utilizadas para minimizar a carência alimentar durante o período crítico para ovinos. Na mesma linha de pensamento, Cunha et al.

\footnotetext{
1 Parte da Dissertação apresentada pelo primeiro autor à UFSM.

2 Médico Veterinário, Mestre em Zootecnia na UFSM.

3 Médico Veterinário, Prof. Dr. do Depto Zootecnia da UFSM. E.mail: cpires@ccr.ufsm.br

4 Aluna do curso de Zootecnia da UFSM. Bolsista da FAPERGS.

5 Engenheiro-Agrônomo, Professor Dr. do Departamento de Zootecnia da UFSM.

6 Aluno do curso de Zootecnia da UFSM.
} 
(2000) descrevem que a utilização de forragens de alta produtividade e alto valor nutritivo é considerada uma alternativa viável para o desenvolvimento da ovinocultura do Nordeste.

De acordo com Neres et al. (2001), nas pastagens nativas, dificilmente obtém-se boa produtividade e qualidade de carne ovina, devido principalmente à deficiência de nutrientes, havendo necessidade da utilização de pastagens cultivadas, suplementação em pastejo e/ou confinamento para explorar o máximo potencial genético dos animais.

Segundo Sampaio et al. (2001), a técnica de alimentação suplementar, denominada creep feeding, pode tornar-se quesito indispensável, para encurtar o tempo necessário ao acabamento dos animais para o abate, além de proporcionar significativo descanso da matriz, melhorando suas funções reprodutivas. De acordo com Carvalho (1998), a utilização do confinamento permite atender com maior facilidade as exigências nutricionais dos animais, possibilitando a terminação de ovinos em períodos de carência alimentar ou quando as pastagens ainda não estejam prontas, além de disponibilizar, no mercado, carne ovina de qualidade no período de entre safra, quando são obtidos os melhores preços.

A carcaça, por apresentar a porção comestível, é o elemento mais importante do animal; portanto, devem ser comparadas suas características para serem verificadas as diferenças existentes entre raças ou cruzamentos, procurando animais que produzam melhores carcaças, o que beneficiará a comercialização (Loose et al., 1981).

Alcade (1990) ressalta a importância de conhecer qual a proporção do animal vivo que será convertido em carcaça, isto é, o "rendimento", conceituado como o percentual do peso de carcaça em relação ao peso vivo de abate. Segundo Sañudo \& Sierra (1993), o rendimento de carcaça é a percentagem de peso da carcaça obtida em relação a determinado peso vivo, que pode ser influenciado por fatores intrínsecos ao animal e por fatores extrínsecos ao sistema de alimentação e manejo imposto aos animais

Macedo et al. (2000) descrevem que a gordura é o componente da carcaça que apresenta maior variação, influenciada principalmente pelo sistema de terminação, pelo genótipo e pela razão idade/peso do animal, sendo uma questão fundamental, pois existe a aversão do consumidor moderno pelo excesso de tecido adiposo.

O trabalho foi conduzido com o objetivo de avaliar o desempenho dos animais e as características da carcaça de cordeiros terminados em pastagem natural suplementada, pastagem cultivada de azevém (Lolium multiflorum Lam.) e confinamento.

\section{Material e Métodos}

O experimento foi conduzido no Departamento de Zootecnia da Universidade Federal de Santa Maria (UFSM), situada na Depressão Central do Rio Grande do Sul, com altitude de 95 metros, latitude 29 $403^{\prime} \mathrm{Sul}$ e longitude 53\%42' Oeste (Araújo, 1930). O solo pertence à unidade de mapeamento São Pedro, classificado com Podzólico Vermelho Amarelo Distrófico, que se caracteriza por ser profundo, avermelhado, com textura superficial arenosa (Brasil, 1973).

O período experimental correspondeu aos meses de julho a novembro de 2000. De acordo com a Estação Meteorológica de Santa Maria, nesse período, as médias mensais foram: temperatura $17,88^{\circ} \mathrm{C}$, umidade relativa do ar 78,80\%; e precipitação de $165,08 \mathrm{~mm}$. A precipitação total no período do experimento foi de $825,40 \mathrm{~mm}$.

Foram utilizados 16 cordeiros cruzas Ile de France $\mathrm{x}$ Texel, de parto simples, distribuídos aleatoriamente com suas respectivas mães, nos tratamentos: PNSPastagem natural suplementada PC- Pastagem cultivada de azevém (Lolium multiflorum Lam) e CONConfinamento.

A pastagem natural com área de 1,10 ha, roçada e diferida 60 dias antes do início do experimento, foi subdividida em duas áreas menores e, por intermédio da estimativa da composição botânica descrita por Tothill et al. (1978), estava constituída pelas espécies Cynodon dactylon (25,58\%), Eragrostis plana (23,57\%), Paspalum notatum (20,83\%), Cyperus sp. (15,18\%), Eragrostis lugens (10,30\%), Axonopus affinis (2,91\%), Desmodium sp. (0,39\%), Orthopapus angustifolis (0,38\%), Soliva sp $(0,34 \%)$, Eleusine tristachya $(0,24 \%)$ e Scutelaria racemosa $(0,15 \%)$.

A pastagem cultivada de azevém (Lolium multiflorum Lam.), com área total de 1,35 ha, foi subdividida em duas áreas menores e estabelecida no dia 15 de maio, com $50 \mathrm{~kg} / \mathrm{ha}$ de semente, com uma adubação de base de $200 \mathrm{~kg} / \mathrm{ha}$ de NPK (05-20-20) e adubação nitrogenada de $90 \mathrm{~kg} / \mathrm{ha}$ de nitrogênio dividida em três aplicações.

A massa de forragem da pastagem natural e cultivada foi avaliada a cada 21 dias. As avaliações foram realizadas pelo método de dupla amostragem, conforme Gardner (1986). A massa de forragem foi

R. Bras. Zootec., v.33, n.1, p.225-233, 2004 
expressa em $\mathrm{kg} / \mathrm{ha}$ de MS, em que os valores médios durante o experimento foram na pastagem cultivada e pastagem natural de 1320 e 1770 , respectivamente. No dia correspondente à metade do intervalo das duas avaliações, foi coletada por dois avaliadores a forragem aparentemente consumida pelos animais por intermédio da técnica de simulação de pastejo (Gibb \& Treacher, 1976).

$\mathrm{Na}$ massa de forragem avaliada na dupla amostragem, foi realizada a separação manual do material senescente do material verde. Os valores de material verde seco, em kg/ha de MS, foram de 869 na pastagem cultivada e 673 na pastagem natural.

A oferta de forragem verde foi expressa em $\mathrm{kg}$ de MS/100 kg de PV, sendo de 9,16 para a pastagem cultivada e 6,72 para a pastagem natural. O sistema de pastejo utilizando foi o contínuo. Foi realizada a estimativa da taxa de acúmulo de matéria seca, utilizando gaiolas de exclusão, e para o cálculo da taxa de acúmulo foi utilizada a equação descrita por Campbell (1966).

No confinamento a céu aberto (área de $7 \mathrm{~m}^{2} /$ animal) com piso forrado com casca de arroz, a silagem de sorgo (AG 2002) foi fornecida à vontade, às 8 e $16 \mathrm{~h}$, e a quantidade oferecida, ajustada diariamente, aumentado ou diminuindo em $10 \%$, conforme a sobra do dia anterior, que deveria ser de até $20 \%$ da quantidade oferecida.

O concentrado utilizado para os animais da pastagem natural e confinamento foi fornecido pela Santista Alimentos, onde foi oferecido ao conjunto ovelha mais cordeiro até a idade média dos 42 dias dos cordeiros, em uma quantidade equivalente a 1,3\% do peso vivo/dia. Após esse período, o concentrado foi ofertado às ovelhas em quantidade equivalente a $0,5 \%$ do peso vivo e aos cordeiros, a $2 \%$ do peso vivo, de forma privativa (creep feeding) até atingirem o peso de abate.

O controle de endoparasitos dos animais foi realizado com a contagem de ovos por gramas de fezes (OPG).

Os animais, ovelhas e cordeiros, foram pesados no início do experimento, e a cada intervalo de 21 dias até o abate com jejum de sólidos por 14 horas.

As variáveis avaliadas nos cordeiros foram peso ao nascimento, peso do início do experimento, peso de abate, ganho médio diário, dias do cordeiro no experimento para atingir o peso de abate. Após o abate, foram obtidos o peso e o rendimento da carcaça quente. Após 24 horas de resfriamento da carcaça a $2^{\circ} \mathrm{C}$, foram obtidos o peso e o rendimento de carcaça fria, índice de quebra ao resfriamento. A área de olho de lombo foi obtida pela exposição do músculo
Longíssimus dorsi após um corte transversal na carcaça, entre a $12^{\mathrm{a}}$ e $13^{\mathrm{a}}$ costela, traçando o seu contorno em papel vegetal (Müller, 1980). Para determinação e registro da área, foi utilizado o programa SITER 3.1 modelo A 2 descrito por Giotto (2001). Nesta seção, toma-se a espessura da gordura de cobertura com o uso de um paquímetro e o marmoreio $(1.0=$ não existente até $5,0=$ existente $)$. Na metade esquerda da carcaça, foi tomado o comprimento de carcaça, distância entre o bordo anterior da sínfise ísquio-pubiana e o bordo anterior da primeira costela no seu ponto médio, compacidade da carcaça (peso da carcaça fria/comprimento interno da carcaça); comprimento de perna, distância entre o bordo anterior da sínfise ísquio-pubiana e a porção média dos ossos do tarso; profundidade de peito, distância máxima entre o dorso e o externo; espessura de coxão, distância entre os bordos interno e externo da parte superior da perna em sua parte mais larga e gordura de cobertura $(1,0=$ excessivamente magra até $5,0=$ excessivamente gorda), estas avaliações seguiram a técnica descrita por Osório (1998).

Para análise estatística dos dados, foi adotado o delineamento experimental inteiramente casualizado com três tratamentos e número variável de repetições. A metodologia estatística incluiu a análise de variância e teste F. Diferenças significativas em nível de 5\% ensejaram aplicação do teste Tukey neste mesmo nível de significância. Foram feitos estudos de regressão do ganho médio diário em relação ao tempo em dias no experimento. As análises foram realizadas utilizando o pacote estatístico SAS (SAS, 1993).

\section{Resultados e Discussão}

Na Tabela 1, estão os valores do peso ao nascer, peso início do experimento, peso de abate, ganho de peso médio diário e dias do cordeiro no experimento para atingir o peso de abate, nos diferentes tratamentos.

Os bons pesos ao nascer devem-se ao padrão zootécnico dos animais e às boas condições nutricionais das ovelhas no terço final da gestação. De acordo com Coimbra Filho (1992), o peso ao nascimento está diretamente relacionado com fatores de ordem genética, idade e nutrição das ovelhas, além do sexo e número de cordeiros nascidos. Pires et al. (2000) verificaram em animais da raça Texel pesos médios de 4,9 $\mathrm{kg}$ às 24 horas após o nascimento de cordeiros de parto simples, resultados semelhantes aos do presente trabalho.

O maior peso no início do experimento dos ani-

R. Bras. Zootec., v.33, n.1, p.225-233, 2004 
mais do confinamento não interferiu nos resultados obtidos, pois, em uma análise preliminar utilizando o peso como covariável, os resultados não se alteraram.

$\mathrm{O}$ peso ao abate em torno de $31 \mathrm{~kg}$ foi préestabelecido no início do experimento. Figueiró (1989) descreve que a idade de abate dos cordeiros deve ser entre 90 e 100 dias, com peso vivo de 25 a $30 \mathrm{~kg}$ ao abate. Para satisfazer o consumidor, Osório et al. (1998) descrevem que existe um peso ótimo para sacrifício dos animais, em que a proporção de músculo é máxima; a de osso, mínima; e a de gordura, suficiente para proporcionar à carcaça as propriedades de conservação e a carne suas propriedades sensoriais exigidas.

Siqueira (2000), ao comparar pesos de abate (28, 32,36 e $40 \mathrm{~kg}$ ), qualidade da carcaça e renda líquida por cordeiro, concluiu que, sob o ponto de vista econômico, o peso de abate de $28 \mathrm{~kg}$ é o melhor. Para qualidade da carcaça, os pesos de 28,32 e $36 \mathrm{~kg}$ foram similares, ao passo que $40 \mathrm{~kg}$ resultou em carcaças com teores de gordura muito elevados.

O ganho médio diário (GMD) dos cordeiros da pastagem cultivada foi superior aos demais tratamentos, devido ao valor nutritivo da pastagem ingerida (Tabela 2), adequada oferta de material verde na pastagem, o que não restringiu o consumo dos animais. Outro fator que provavelmente contribuiu para o desempenho dos cordeiros foi a quantidade leite ingerido em função da produção do leite das ovelhas que, além de produzirem leite, tiveram GMD de $0,081 \mathrm{~kg} / \mathrm{dia}$.

Silva Sobrinho (1996) descreve que as ovelhas normalmente perdem peso no início da lactação, o que não ocorreu no presente tratamento, portanto, isto deve ter refletido na quantidade de leite disponível e ao elevado (GMD) dos cordeiros. A superioridade no desempenho de cordeiros em pastagem cultivada em relação à pastagem natural foi descrita por Ávila \& Osório (1996), ao avaliarem sistemas de terminação de cordeiros, onde encontraram ganhos médios diários superiores para cordeiros criados em pastagem cultivada de aveia, azevém e trevo branco, quando comparados aos criados em pastagem nativa $(0,252$ e $0,179 \mathrm{~kg} / \mathrm{dia})$.

Canto et al. (1999), ao manterem resíduos de MS em níveis superiores a $2400 \mathrm{~kg}$ de MS por ha, encontraram desempenho animal de $0,122 \mathrm{~kg} / \mathrm{dia}$, resultados inferiores ao presente experimento.

$\mathrm{Na}$ Tabela 2, estão os percentuais de matéria de seca, matéria mineral, digestibilidade in vitro da matéria seca, nutrientes digestíveis totais, proteína bruta, da silagem de sorgo, concentrado, simulação de pastejo da pastagem natural, simulação de pastejo da pastagem cultivada.

Os resultados encontrados na Tabela 2 mostram o maior valor protéico da simulação de pastejo da pastagem natural em relação à silagem de sorgo e valores aproximados para o percentual de nutrientes digestíveis totais, o que pode justificar o maior GMD dos animais da PNS em relação aos animais do CON, embora a oferta de concentrado tenha sido na mesma proporção para os dois tratamentos. Banchero \& Montossi (1998), ao avaliarem níveis de oferta de forragem (trevo alexandrino e azevém tetraplóide) e suplementação com $3 \%$ do peso vivo de farelo de trigo em cordeiros desmamados precocemente, encontraram ganhos médios diários de $0,047 \mathrm{~kg}$ dos 78 aos 150 dias. Julián et al. (1998) encontraram ganhos médios diários de $0,104 \mathrm{~kg}$ para cordeiras de 9-10 meses de idade, suplementadas em pastagem natural com $1,3 \%$ do peso vivo com farelo de trigo.

Suplementando borregos desmamados aos $20 \mathrm{~kg}$, com concentrado (16\% PB e $70 \%$ NDT) na proporção de $1 \% \mathrm{PV}$, com valores nutricionais em pastagem de Cynodon spp. cv. Tifton-85 (10,57\% PB), Oliveira

Tabela 1 - Valores médios para peso ao nascimento $(\mathrm{PN})$, peso do início do experimento (PIT), ganho médio diário (GMD), dias do cordeiro no experimento para atingir o peso de abate, (TE), mos diferentes tratamentos, coeficiente de variação $(\mathrm{CV})$, valor de $F(F)$ e probabilidade $(\mathrm{Pr}>\mathrm{F})$

Table 1 - Means for birth weight (WB), weight at the beginning of the experiment (WBE), average daily gain (DWG), days for the animal to achieve the slaughter weight, $(F I)$, in different treatments, coefficient of variation (CC), $F$ value $(F)$ and probability (Pr>F)

\begin{tabular}{lcccccc}
\hline \multicolumn{7}{c}{ Tratamentos } \\
& \multicolumn{7}{c}{ Treatments } & & & \\
\cline { 2 - 5 } Variáveis & PNS & PC & CON & CV & F & Pr $>$ F \\
Variables & $N S P$ & $R P$ & $C O N$ & $C C$ & $F$ & $P r>F$ \\
\hline PN $(\mathrm{kg})$ & $5,02^{\mathrm{a}}$ & $4,67^{\mathrm{a}}$ & $5,11^{\mathrm{a}}$ & 13,15 & 1,11 & 0,3593 \\
$W B(\mathrm{~kg})$ & & & & & & \\
PIT $(\mathrm{kg})$ & $6,91^{\mathrm{b}}$ & $6,27^{\mathrm{b}}$ & $10,37^{\mathrm{a}}$ & 18,69 & 11,15 & 0,0015 \\
$W B E(\mathrm{~kg})$ & & & & & & \\
GMD $(\mathrm{kg})$ & $0,32^{\mathrm{b}}$ & $0,40^{\mathrm{a}}$ & $0,21^{\mathrm{c}}$ & 8,51 & 56,81 & 0,0001 \\
$D W G(\mathrm{~kg})$ & & & & & & \\
TE (dias) & $75,67^{\mathrm{b}}$ & $62,00^{\mathrm{b}}$ & $98,50^{\mathrm{a}}$ & 11,45 & 21,01 & 0,0001 \\
$F I($ days $)$ & & & & & & \\
\hline
\end{tabular}

Médias seguidas de letras diferentes na linha diferem $(\mathrm{P}<0,05)$ pelo teste Tukey.

PNS - pastagem natural suplementada, PC - pastagem cultivada de azevém e CON - confinamento.

Means followed by different letters in the line differ $(P<.05)$ by Tukey test NSP - natural supplemented pasture; RP - ryegrass pasture and CON confinement. 
et al. (2001) observaram ganhos médios diários de $0,133 \mathrm{~kg}$ dos 99 aos 178 dias de idade dos animais. Os resultados descritos nos experimentos anteriores foram inferiores aos do presente trabalho.

A limitação do concentrado oferecido as ovelhas e aos cordeiros no creep feeding, associada ao baixo valor nutritivo e à digestibilidade do volumoso, também foi um fator limitante no consumo, onde os animais ingeriram $1,5 \% \mathrm{de} \mathrm{MS} / \mathrm{kg}$. Isto determinou o baixo ganho médio diário dos cordeiros no confinamento, o que também pode ser justificado pela diminuição do estado corporal das ovelhas que perderam, em média, $0,12 \mathrm{~kg} /$ dia durante o experimento, o que refletiu em menor produção de leite aos cordeiros. Outro fator que pode ter influenciado foi o tipo de confinamento a céu aberto e suas condições ambientais, o que abre caminhos para novas pesquisas para utilização deste sistema em nossas condições ambientais. Quando a alimentação oferecida aos animais em confinamento se aproxima das necessidades nutricionais, podem ser obtidos melhores resultados para o ganho médio diário. Kafedjiev et al. (1998) encontraram ganho médio diário de $0,216 \mathrm{~kg}$, ao confinar cordeiros da raça Tsigai com feno tratado com amônia (9,85\% PB). Já Silva (1999) obteve valores para ganho médio diário de $0,245 \mathrm{~kg}$ do nascimento ao abate 28 ou $30 \mathrm{~kg}$, quando alimentou os animais em um sistema de confinamento coberto de piso ripado e atendendo as exigências nutricionais dos animais. Motta (2000) também observou GMD de $0,185 \mathrm{~kg} / \mathrm{dia}$ em cordeiros alimentados em piso de cimento e totalmente coberto, com silagem de milho ( $8 \% \mathrm{~PB})$, para o conjunto (ovelha + cordeiro) e fornecimento de concentrado $(20 \% \mathrm{~PB})$ apenas aos cordeiros creep feeding do nascimento ao desmame aos 60 dias de idade.

Ganhos elevados foram obtidos por Bueno et al. (1998), ao avaliarem o desempenho de cordeiros Suffolk alimentados com diferentes tipos de volumoso, em que os animais foram alimentados com silagem de milho $(7,6 \% \mathrm{~PB})$ à vontade, mais concentrado $(20 \%$ $\mathrm{PB})$ na quantidade de $3,5 \%$ peso vivo, obtendo-se ganho médio diário de $0,320 \mathrm{~kg}$.

Na Figura 1, estão dispostos o ganho médio diário, em função do tempo em dias em três sistemas de alimentação, e suas respectivas equações de regressão.

Verifica-se, na Figura 1, que, à medida que os cordeiros aumentam a idade, o ganho médio diário diminui, o que pode ser explicado pela dependência dos cordeiros do leite materno nas primeiras semanas de lactação. Siqueira et al.(1998) descrevem que o pico da produção de leite ocorre entre a $3^{\mathrm{a}}$ e $4^{\mathrm{a}}$ semanas após o parto, sendo que $75 \%$ do total da lactação é produzido nas primeiras oito semanas e, após o ganho de peso dos cordeiros, depende, principalmente, da alimentação sólida.

As diferenças entre os tratamentos demonstradas na Figura 1 podem ser explicadas pelo desempenho das ovelhas para o GMD, em kg, PNS $(-0,680)$, PC $(0,081)$ e CON $(-0,120)$, demonstrando que melhor

Tabela 2 - Percentuais de matéria seca (MS) e matéria mineral (MM), digestibilidade in vitro da matéria seca (DIVMS), nutrientes digestíveis totais (NDT), proteína bruta (PB), da silagem de sorgo (SS), concentrado (CC), forragem aparentemente consumida na pastagem natural (FCPN), forragem aparentemente consumida na pastagem cultivada (FCPC)

Table 2 - Percentage of dry mater (DM) and mineral mater (MM), in vitro dry mater digestibility (VDDM), total digestible nutrient (TDN) and crude protein (CP) of sorghum silage (SS), concentrate (CC), forage apparently consumed in the natural pasture (FACNP) and forage apparently consumed in the ryegrass pasture (FACRP)

\begin{tabular}{lccccc}
\hline Componentes da dieta & MS & MM & DIVMS & NDT & PB \\
Diet components & $D M$ & $M M$ & $I V D D M$ & $T D N$ & $C P$ \\
\hline SS & 24,35 & 4,53 & 43,25 & 41,77 & 6,54 \\
SS & & & & & \\
CC & $87,00^{*}$ & 7,59 & 67,28 & 65,82 & 17,72 \\
$C C$ & & & & 39,14 & 13,27 \\
FCPN & 31,43 & 7,72 & 41,68 & 62,81 & 19,99 \\
FACNP & 18,35 & 9,89 & 76,31 & & \\
FCPC & & & &
\end{tabular}

* Dado indicado pelo fornecedor.

* Value indicated by the supplier. 
desempenho das ovelhas ocasionou maiores GMD e produção de leite aos cordeiros. Susin et al. (1996) descreveram que a nutrição da ovelha, durante o início da lactação, interfere na produção de leite e no peso ao desmame do cordeiro e, quando é deficiente, obriga a ovelha a utilizar suas reservas, perdendo peso na lactação.

A importância da produção de leite no desempenho dos cordeiros no presente trabalho corroboram os resultados de Carvalho et al. (1999), os quais observaram redução no ganho médio diário após o desmame de $0,309 \mathrm{~kg}$ para $0,145 \mathrm{~kg}$.

$\mathrm{Na}$ Tabela 3, estão os pesos de carcaça quente e fria, índice de quebra ao resfriamento, rendimento de carcaça quente e fria em três sistemas de alimentação. Os resultados mostram que não há diferença entre os animais da PC e PNS para peso de carcaça quente e peso de carcaça fria, porém foram superiores aos animais do confinamento, embora todos os cordeiros tenham sido abatidos praticamente ao mesmo peso.

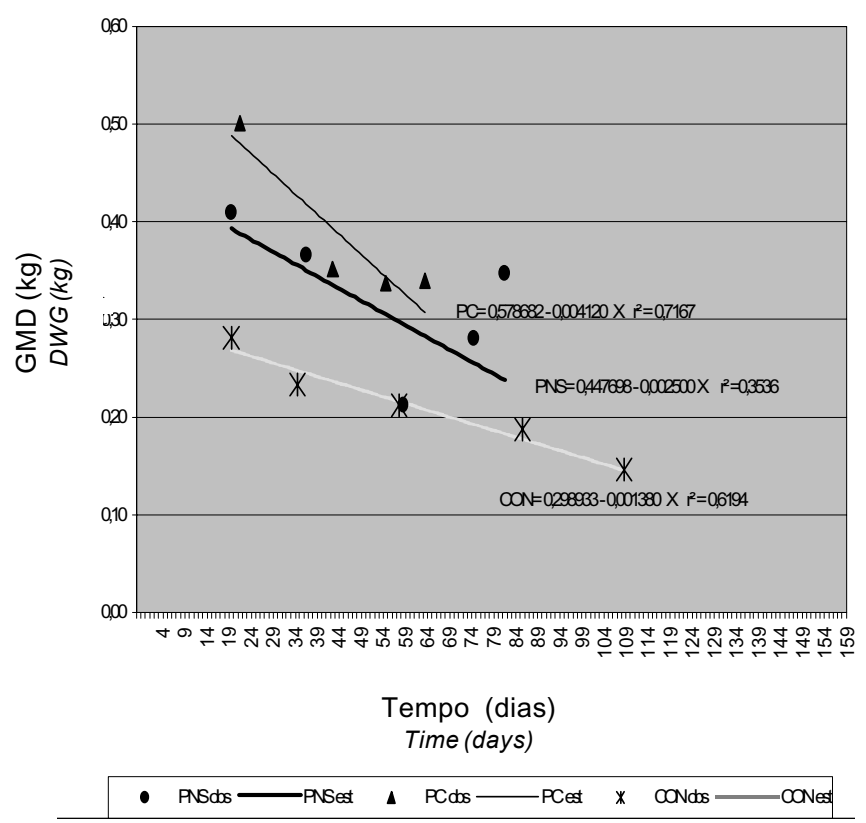

Figura 1 - Ganho médio diário $(\mathrm{kg})$ em relação ao tempo de experimento (dias), de cordeiros terminados em: PNS - Pastagem natural suplementada, PC - Pastagem cultivada de azevém (Lolium multiflorum Lam.), CON - Confinamento.

Figure 1 - Average daily gain $(\mathrm{kg})$ in relation to the experimental time (days), from finished lambs in: NSP - Natural supplemented pasture, GP - Ryegrass pasture (Lolium multiflorum Lam.), CON - Confinement.
Estes resultados devem-se ao maior ganho médio diário obtidos pelos cordeiros das pastagens cultivadas e naturais. Sainz (1996) observa que o peso da carcaça é influenciado pela velocidade de crescimento, idade ao abate e regime nutricional dos animais. Motta (2000), também comparando três métodos de alimentação, sexo e peso de abate (25 e $33 \mathrm{~kg})$, encontrou diferença significativa $(\mathrm{P}<0,05)$ para peso de carcaça quente, demonstrando que, para melhor nível nutricional do nascimento ao desmame, aos 60 dias, corresponde a maior peso de carcaça.

O índice de quebra ao resfriamento foi igual entre os tratamentos, embora os animais da pastagem cultivada tenham apresentado maior teor de gordura na carcaça. Carneiro (2001) encontrou valores próximos ao presente experimento ao comparar o índice de quebra ao resfriamento da carcaça ao abater cordeiros de parto simples desmamados e não desmamados e cordeiros de parto duplo desmamados, não encontrando diferença $(\mathrm{P}>0,05)$ entre os tratamentos, cujos valores foram de 2,$75 ; 2,29$ e $2,98 \%$ respectivamente. Osorio et al. (1996) encontraram maiores índices de quebra ao resfriamento em relação ao presente experimento, cujos valores foram de 3,83\% para cordeiros da raça Texel, comparando cinco grupos genéticos das raças Merina, Ideal, Corriedale e Romney Marsh, abatidos aos 225 dias de idade.

$\mathrm{O}$ rendimento de carcaça fria $(52,537 \%)$ dos animais da pastagem cultivada foi superior $(\mathrm{P}<0,05)$ aos demais tratamentos, devido, principalmente, ao menor conteúdo gastrintestinal e à maior deposição de gordura na carcaça, em razão do melhor nível nutricional dos animais. Quanto à gordura, a afirmação é respaldada por Pereira et al. (2001), que, ao avaliarem o efeito da castração sobre a morfologia e características produtivas e comerciais em cordeiros castrados e não castrados com 123 dias de idade, com peso vivo em torno dos $29 \mathrm{~kg}$, encontraram maiores rendimentos de carcaça para os animais castrados, devido à maior quantidade de gordura na carcaça.

Na Tabela 4, estão os valores médios em cada tratamento para comprimento da carcaça, comprimento de perna, compacidade, espessura de coxão, profundidade do peito, área de lombo, espessura de gordura, gordura de cobertura e marmoreio em cordeiros em três sistemas de alimentação.

O comprimento da carcaça e comprimento da perna foram semelhantes entre os tratamentos $(\mathrm{P}>0,05)$. Resultados aproximados aos do presente experimento foram obtidos por Motta (2000), ao 
comparar três métodos de alimentação, sexo e peso ao abate de $33 \mathrm{~kg}$, que encontrou valores médios para comprimento da carcaça e comprimento da perna 54,65 e $34,46 \mathrm{~cm}$, respectivamente.

Os resultados da compacidade da carcaça mostram que os cordeiros da PNS e PC são semelhantes entre si e superiores aos animais do CON. Bueno et al (2000), ao avaliarem a carcaça de cordeiros Suffolk abatidos em diferentes idades, descrevem que a compacidade mostrou acréscimo linear positivo com o aumento do peso da carcaça, o que concorda com o presente experimento, onde as carcaças mais pesadas foram da PNS e PC. Resultados semelhantes aos dos presente experimento também foram obtidos por Zundt et al. (2001), em que foram obtidos valores de $0,271 \mathrm{~kg} / \mathrm{cm}$ para a compacidade, ao avaliarem diferentes níveis protéicos em cordeiros pesando em torno de $29 \mathrm{~kg}$ ao abate.

A área de olho de lombo mostrou semelhança entre os tratamentos, embora os animais do confinamento tenham apresentado menor valor $\mathrm{em}^{2} \mathrm{~cm}^{2}$, devido ao menor peso de carcaça quente. Para esta mesma

Tabela 3 - Valores médios, para peso carcaça quente (PCQ), peso carcaça fria (PCF), índice de quebra ao resfriamento (IQ), rendimento carcaça fria (RCF), rendimento carcaça quente (RCQ), nos diferentes tratamentos, valor de $F$ $(F)$, coeficiente de variação $(C V)$ e probabilidade $(\mathrm{Pr}>\mathrm{F})$

Table 3 - Means values for hot carcass weight (HCW), cold carcass weight $(C C W)$, cooling index $(B I C)$, cold carcass yield (CCY), hot carcass yield ( $\mathrm{HCY}$ ), in the different treatments, $F$ value $(F)$, coefficient of variation (CV) and probability $(\mathrm{Pr}>\mathrm{F})$

\begin{tabular}{|c|c|c|c|c|c|c|}
\hline \multirow[b]{2}{*}{$\begin{array}{l}\text { Variáveis } \\
\text { Variables }\end{array}$} & \multicolumn{3}{|c|}{$\begin{array}{c}\text { Tratamentos } \\
\text { Treatments }\end{array}$} & \multirow[b]{2}{*}{$\mathrm{CV}$} & \multirow[b]{2}{*}{$\mathrm{F}$} & \multirow[b]{2}{*}{$\operatorname{Pr}>\mathrm{F}$} \\
\hline & $\begin{array}{l}\text { PNS } \\
N S P\end{array}$ & $\begin{array}{l}\mathrm{PC} \\
R P\end{array}$ & $\begin{array}{l}\mathrm{CON} \\
\mathrm{CON}\end{array}$ & & & \\
\hline$\overline{\mathrm{PCQ}(\mathrm{kg})}$ & $15,73^{\mathrm{a}}$ & $16,81^{\mathrm{a}}$ & $14,28^{b}$ & 4,80 & 13,35 & 0,0007 \\
\hline PCF (kg) & $15,36^{\mathrm{a}}$ & $16,46^{\mathrm{a}}$ & $13,93^{b}$ & 4,94 & 13,28 & 0,0007 \\
\hline $\begin{array}{l}C C W \\
\mathrm{IQ}(\% \\
B I C(\%)\end{array}$ & + & & $2,52^{\mathrm{a}}$ & 5,80 & 1,43 & 2749 \\
\hline $\begin{array}{l}\text { RCQ }(\%) \\
H C Y(\%)\end{array}$ & $50,24^{\mathrm{a}}$ & $53,66^{\mathrm{a}}$ & $45,92^{\mathrm{b}}$ & 4,23 & 15,89 & 0003 \\
\hline $\begin{array}{l}\mathrm{RCF}(\%) \\
C C Y(\%)\end{array}$ & $49,07^{\mathrm{b}}$ & $52,54^{\mathrm{a}}$ & $44,77^{\mathrm{c}}$ & 4,25 & 16,56 & 0,0003 \\
\hline
\end{tabular}

Médias seguidas de letras diferentes na linha diferem $(P<0,05)$ pelo teste Tukey.

PNS - pastagem natural suplementada, PC - pastagem cultivada de azevém e CON - confinamento.

Means followed by different letters in the line differ $(P<.05)$ by Tukey test $N S P$ - natural supplemented pasture; RP - ryegrass pasture and CON confinement.

R. Bras. Zootec., v.33, n.1, p.225-233, 2004 variável, Carvalho (1998) não encontrou diferença $(\mathrm{P}>0,05)$ entre cordeiros machos inteiros $(11,21$ $\left.\mathrm{cm}^{2}\right)$ e machos castrados $\left(11,38 \mathrm{~cm}^{2}\right)$. Osório et al. (1999), ao avaliarem a produção de carne entre cordeiros castrados e não castrados abatidos com peso vivo de 27,53 e $30,64 \mathrm{~kg}$, respectivamente, demostraram para área de olho de lombo valores de 10,2 e $11,99 \mathrm{~cm}^{2}$, respectivamente, portanto, inferiores ao encontrados nos animais da PC e PNS.

A espessura de gordura mostrou que os animais da PC apresentaram valores superiores à PNS e $\mathrm{CON}$, embora que entre estes os resultados sejam semelhantes. O maior valor da espessura de gordura dos animais da PC é explicado pela maior deposição de gordura na região da costela destes animais, em função do maior ganho médio diário. Estes resultados são corroborados por Silva (1999), ao avaliar o crescimento, composição corporal e exigências nutricionais de cordeiros abatidos com diferentes pesos, quando demonstrou que a proporção de godura aumentou do nascimento ao desmame, causado pela alimentação dos cordeiros juntamente com a mãe além do leite materno. Comparando a espessura de gordura com a gordura de cobertura, embora os critérios de avaliações sejam diferentes, deveriam mostrar mais uniformidade entre as variáveis. A possível explicação dos resultados seria que os animais da pastagem cultivada tiveram deposição de gordura mais localizada no lombo, onde é medida a espessura de gordura, contudo, para a gordura de cobertura, onde foi avaliada a distribuição da gordura em toda a carcaça, foi mais uniforme tornando mais próximos os resultados entre os tratamentos.

Kempster et al. (1987) descrevem que a deposição de gordura na carcaça é de forma rápida pelo lombo e costilhar, após crescimento mais lento intermuscular e pela região da paleta, o que concorda com a forma de deposição da gordura no presente experimento.

Para cobertura de gordura, os animais da PC foram semelhantes aos animais da PNS e superiores ao $\mathrm{CON}$, contudo entre estes não apresentan diferença significativa $(\mathrm{P}>0,05)$. Resultados inferiores foram encontrados por Macedo et al. (2000)m ao descreverem que os cordeiros terminados em pastagem de Coastcross (Cynodon dactylon) tiveram menor valor para cobertura de gordura em suas carcaças (média de 2,43), comparando aos terminados em confinamento $(2,95)(\mathrm{P}<0,05)$, corroborando os resultados de Arnold \& Meyer (1988). 
Tabela 4 - Valores médios para comprimento da carcaça (CC), comprimento de perna (CP), compacidade (CM), espessura de coxão (EC), profundidade de peito (PRP), espessura de gordura $(E G)$, marmoreio (MAR), área de lombo (Al) e gordura de cobertura (GC), nos diferentes tratamentos, valor de $F(F)$, coeficiente de variação (CV) e probabilidade (Pr>F)

Table 4 - Mean values of carcass length (CL), leg length (LL), compactiveness (CM), thigh thickness (TT), chest deepness (CD), loin area (LA), fat thickness (GT), fat cover (CG) and marbling (MAR) for the different treatments, $F$ value $(F)$, coefficient of variation $(\mathrm{CV})$ and probability $(\mathrm{Pr}>\mathrm{F})$

\begin{tabular}{|c|c|c|c|c|c|c|}
\hline \multirow{3}{*}{$\begin{array}{l}\text { Variáveis } \\
\text { Variables }\end{array}$} & \multicolumn{3}{|c|}{$\begin{array}{c}\text { Tratamentos } \\
\text { Treatments }\end{array}$} & \multirow{3}{*}{ CV } & & \multirow{3}{*}{$\operatorname{Pr}>\mathrm{F}$} \\
\hline & PNS & $\mathrm{PC}$ & $\mathrm{CON}$ & & & \\
\hline & $N S P$ & & $\mathrm{CON}$ & & & \\
\hline $\begin{array}{l}\mathrm{CC}(\mathrm{cm}) \\
C L(\mathrm{~cm})\end{array}$ & $54,67^{\mathrm{a}}$ & $54,67^{\mathrm{a}}$ & $56,37^{\mathrm{a}}$ & ,61 & 11 & 160 \\
\hline $\mathrm{P}(\mathrm{cm})$ & $32,33^{\mathrm{a}}$ & $31,80^{\mathrm{a}}$ & $32,62^{\mathrm{a}}$ & 3,18 & 0,85 & 0,4485 \\
\hline$M$ & $0,29^{\mathrm{a}}$ & $0,31^{\mathrm{a}}$ & $0,25^{\mathrm{b}}$ & 5,34 & 14,97 & 0,0004 \\
\hline $\begin{array}{l}\mathrm{EC}(\mathrm{cm}) \\
C T(\mathrm{~cm})\end{array}$ & $9,12^{\mathrm{a}}$ & $9,03^{\mathrm{a}}$ & $9,62^{\mathrm{a}}$ & 11,98 & 36 & 0,7047 \\
\hline $\begin{array}{l}\operatorname{PRP}(\mathrm{cm}) \\
C D(\mathrm{~cm})\end{array}$ & $23,92^{\mathrm{a}}$ & $24,07^{\mathrm{a}}$ & $24,25^{\mathrm{a}}$ & 2,24 & 0,46 & 0,6418 \\
\hline $1(\mathrm{c}$ & $14,25^{\mathrm{a}}$ & $14,28^{\mathrm{a}}$ & $11,92^{\mathrm{a}}$ & 11,82 & 3,16 & 0,0761 \\
\hline $\begin{array}{l}L A\left(\mathrm{~cm}^{2}\right) \\
\mathrm{EG}(\mathrm{mm})\end{array}$ & $1,58^{\mathrm{b}}$ & $3,33^{\mathrm{a}}$ & $00^{\mathrm{b}}$ & 31,18 & 26 & 02 \\
\hline $\begin{array}{l}G T(m m) \\
\operatorname{GC}(1-5) \\
C G(1-5)\end{array}$ & $3,25^{\mathrm{ab}}$ & $3,83^{\mathrm{a}}$ & $3,00^{\mathrm{b}}$ & 10,64 & 7,23 & 0,0077 \\
\hline $\operatorname{MAR}(\mathrm{mm})$ & $2,42^{\mathrm{a}}$ & $3,08^{\mathrm{a}}$ & $2,13^{\mathrm{a}}$ & 22,94 & 3,54 & 0,0594 \\
\hline
\end{tabular}

$\operatorname{MAR}(1-5)$

Médias seguidas de letras diferentes na linha diferem $(P<0,05)$ pelo teste Tukey.

PNS - pastagem natural suplementada, PC - pastagem cultivada de azevém e CON - confinamento.

Means followed by different letters in the line differ $(P<.05)$ by Tukey test $N S P$ - natural supplemented pasture; RP - ryegrass pasture and CON confinement.

\section{Conclusões}

Cordeiros terminados em pastagem cultivada de azevém, junto com a mãe, possuem desempenho superior e carcaça com maior espessura de gordura.

As características da carcaça (comprimento, comprimento da perna, espessura de coxão, profundidade de peito e área de olho de lombo) são semelhantes, quando os cordeiros são abatidos ao mesmo peso, embora submetidos a diferentes formas de alimentação.

\section{Literatura Citada}

ALCADE, M.J. Producción de carne en la raza Merina: crescimiento y calidad de la canal. Zaragoza: Faculdad de Veterinária: Universidad de Zaragoza, 1990. 192p. Tese de Licenciatura: Universidad de Zaragoza, 1990.

ARNOLD, A.M.; MEYER, H.H. Effects of gender, time of castration, genotype and feeding regimen on lamb growht and carcass fatness. Journal of Animal Science, v.66, p.2468-2475, 1998.

AVILA, V.S.; OSÓRIO, J.C.S. Efeito do sistema de criação, época de nascimento e ano na velocidade de crescimento de cordeiros. Revista Brasileira de Zootecnia, v.25, n.5, p.1007-1016, 1996.

BANCHERO, G., MONTOSSI, F. Engorde intensivo de corderos: uso alternativo de concentrados, ensilajes y/o pasturas mejoradas. Jornada: Ovinos y pasturas. Serie Actividades de Difusión La Estaanzuela, INIA. Uruguay. n.167, 1998.

BRASIL - Ministério da Agricultura. Levantamento de Reconhecimento dos Solos do Estado do Rio Grande do Sul. Recife, Ministério da Agricultura, 1973. 430p. (Boletim Técnico)

BUENO, M.S.; CUNHA, E.A.; SANTOS, L.E. et al. Desempenho e características de carcaça de cordeiros Suffolk alimentados com diferentes tipos de volumosos. In: REUNIÃO ANUAL DA SOCIEDADE BRASILEIRA DE ZOOTECNIA, 35., 1998, Botucatu. Anais... Botucatu: Sociedade Brasileira de Zootecnia, 1998. v. 1, p.206-208.

BUENO, M.S.; CUNHA, E.A.; SANTOS, L.E. et al. Características de carcaça de cordeiros Suffolk abatidos em diferentes idades. Revista Brasileira de Zootecnia, v.29, n.6, p.1803-1810, 2000.

CAMPBELL, A.G. Grazed pastures parameters: I. pasture dry matter production and availability in a stocking rate and grazing management experiment with dairy cows. Journal Agricultural Science, v.67, n.2, p.211-216, 1966.

CANTO, M.W.; MOOJEN, E.L.; CARVALHO, P.C.F. et al. Produção de cordeiros em pastagem de azevém e trevo-branco sob diferentes níveis de resíduos de forragem. Pesquisa Agropecuária Brasileira, v.34, n.2, p.309-316, 1999.

CARNEIRO, R.M. Avaliação do desempenho de cordeiros de parto simples e duplo desmamados e não desmamados, abatidos aos $30 \mathrm{~kg}$. Santa Maria: Universidade Federal de Santa Maria, 2001. 60p. Dissertação (Mestrado em Zootecnia) Universidade Federal de Santa Maria, 2001.

CARVALHO, S. Desempenho, composição corporal e exigências nutricionais de cordeiros machos inteiros, machos castrados e fêmeas alimentadas em confinamento. Santa Maria: Universidade Federal de Santa Maria, 1998. 100p. Dissertação (Mestrado em Zootecnia) - Universidade Federal de Santa Maria, 1998.

CARVALHO, S.; PIRES, C.C.; PERES, J.R.R. et al. Desempenho de cordeiros machos inteiros, machos castrados e fêmeas, alimentados em confinamento. Ciência Rural, v.29, n.1, p.129-133, 1999.

COIMBRA FILHO, A. Técnicas de criação de ovinos. 2.ed. Guaíba: Agropecuária. 1992. 102p.

CUNHA, E.A.; BUENO, M.S.; SANTOS, L.E. Produção ovina em pastagens. In: CONGRESSO NORDESTINO DE PRODUÇÃO ANIMAL, 2., 2000, Teresina. Anais... Teresina: Sociedade Nordestina de Produção Animal, 2000. p.181-190.

FIGUEIRÓ, P.R.P. Manejo alimentar do rebanho ovino. In: SIMPÓSIO PAULISTA DE OVINOCULTURA, 1., 1989, Campinas. Anais... Campinas: Fundação Cargil, 1989. p.22-33. 
GARDNER, A.L. Técnicas de pesquisa em pastagens e aplicabilidade de resultados em sistemas de produção. Brasília: IICA/EMBRAPA-CNPGL, 1986. 197p. (II CA, Série publicações Miscelâneas, 634).

GIBB, M. J.; TREACHER, T.T. The effect of herbage allowance on lambs graazing perennial ryegrass and red clover swards. Journal Agricultural Science, v.86, p.355-365, 1976.

GIOTTO, E. Manual Siter 3.1. Santa Maria: Universidade Federal de Santa Maria, 2001. 187p.

JULiÁN, R.S.; MONTOSSI, F.; BERRETTA, E.J. et al. Alternativas de alimentacion y manejo invernal de la recria ovina en la region de Basalto. Tacuarembo,Uruguay: INIA, Seminário de Actualização en Tecnologias para Basalto. 1998, p.209-227. (Série Técnica, 102)

KAFEDJIEV, V.; IVANOV, I.; USONOV, G. et al. Results from fattening lambs using hay treated wit ammonia. Zhivotnov"dni-Nauki, v.32, p.16-19, 1998. (Supplement 2)

KEMPSTER, A.J.; CROSTON, D.; GUY, D.R. et al. Growth and carcass characteristics of crossbred lambs by ten sire breds, compared at the same estimated carcass subbcutaneous fat proportion. Animal Production, v.44, p.99-106, 1987.

KIRTON, H., FOURIE, P.D., JURY, K.E. Growth of the carcass and non-carcass components of the Southdown and Romney and their cross and some relationship with composition. New Zeland Journal of Agricultural Research, v.15, p.214-227, 1972.

LOOSE, E.M.; JARDIM, P.O.C.; OSÓRIO, J.C.S. et al.. Peso ao nascer e desenvolvimento ponderal de cordeiros Ideal e cruzas Ideal X Texel. In: REUNIÃO ANUAL DA SOCIEDADE BRASILEIRA DE ZOOTECNIA, 18., 1981, Goiânia. Anais... Goiânia: Sociedade Brasileira de Zootecnia, 1981. p.394.

MACEDO, W.S.L.; REIS, J.C.L. Avaliação de pastagens de inverno utilizados com ovinos. Empresa Brasileira de Pesquisa Agropecuária. EMBRAPA. Centro nacional de pesquisa de ovinos - CNPO Bagé- RS, Coletânia das pesquisas forrageiras. v.1 Bagé.1987 p.131-150, Setor de difusão de tecnologia 525p.

MACEDO, F..A.F.; SIQUEIRA, E.R.; MARTINS, E.N. et al. Qualidade de carcaça de cordeiros Corriedale, Bergamácia $\mathrm{x}$ Corriedale e Hampshire Dow x Corriedale terminados em pastagem e confinamento. Revista Brasileira de Zootecnia, v.29, n. 5, p. $1520-1527,2000$.

MOTTA, O.S. Ganho de peso, características da carcaça de cordeiros(a) em diferentes métodos de alimentação, pesos de abate e produção de leite das ovelhas. Santa Maria: Universidade Federal de Santa Maria, 2000. 76p. Dissertação (Mestrado em Zootecnia) - Universidade Federal de Santa Maria, 2000.

MÜLLER, L. Normas para avaliação de carcaças e concurso de carcaças de novilhos. Santa Maria: Universidade Federal de Santa Maria, 1980. 31p.

NERES, M.A.; MONTEIRO, A.L.G.; GARCIA, C.A. et al. Forma física da ração e pesos de abate nas características de carcaça de cordeiros em creep feeding. Revista Brasileira de Zootecnia, v.30, n.3, p.948-954, 2001. (Suplemento 1)

OLIVEIRA, M.E.; ALENCAR, A.L.G.; NASCIMENTO, M.P.S. et al. Recria e terminação de ovinos em pastagem Cynodon spp. cv.Tifton-85 In: REUNIÃO ANUAL DA SOCIEDADE BRASILEIRA DE ZOOTECNIA, 38., 2001, Piracicaba. Anais... Piracicaba: Sociedade Brasileira de Zootecnia, 2001. p.1051-1052.

OSÓRIO, J.C.; OLIVEIRA, N.M.; NUNES, A.P. Produção de carne em ovinos de cinco genótipos. Perdas e morfologia. Ciência Rural, v.26, n.3, p.477-481, 1996.

OSÓRIO, J.C.; ASTIZ, C.S.; OSÓRIO, M.T. et al. Produção de carne ovina, alternativa para o Rio Grande do Sul. Pelotas: Universidade Federal de Pelotas, 1998. 136p.
OSÓRIO, J.C.S.; JARDIM, P.O.S.; PIMENTEL, M. et al. Produção de carne entre cordeiros castrados e não castrados.1. Cruzas Hampshire Down X Corriedale. Ciência Rural, v.29, n.1, p.135-138, 1999.

PEREIRA, P.H.S.; OSÓRIO, J.C.S.; OSÓRIO, M.T. et al. Efeito da castração sobre a morfologia e características produtivas e comerciais em cordeiros. In: REUNIÃO ANUAL DA SOCIEDADE BRASILEIRA DE ZOOTECNIA, 38., 2001 Piracicaba. Anais... Piracicaba: Sociedade Brasileira de Zootecnia, 2001. p.464-465.

PIRES, C.C.; SILVA, L.F.; SCHLICK, F.E. et al. Cria e terminação de cordeiros confinados. Ciência Rural, v.30, n.5, p.875-880, 2000.

SAINZ. R.D. Qualidade das carcaças e da carne ovina e caprina In: REUNIÃO ANUAL DA SOCIEDADE BRASILEIRA DE ZOOTECNIA, 33., 1996, Fortaleza. Anais... Fortaleza: Sociedade Brasileira de Zootecnia, 1996. p.7.

SAÑUDO, C.; SIERRA, I. Calidad de la canal y de la carne en la especie ovina. Ovino y caprino. Madrid: Consejo General de Colegios Veterinarios, 1993. p.207-254.

SAMPAIO, A.A.M.; BRITO, R.M.; ROUTMAN, K.S. et al. Utilização de Nacl no suplemento com alternativa de viabilizar o creep feeding. . In: REUNIÃO ANUAL DA SOCIEDADE BRASILEIRA DE ZOOTECNIA, 38., 2001, Piracicaba. Anais... Piracicaba: Sociedade Brasileira de Zootecnia, 2001. p.987-988.

STATISTICAL ANALYSES SYSTEM- SAS. SAS/SAT user's guide: statistics. version 6.8. Cary: 1993. 956p.

SILVA, L.F. Crescimento, composição corporal e exigências nutricionais de cordeiros abatidos com diferentes pesos. Santa Maria: Universidade Federal de Santa Maria, 1999. 65p. Dissertação (Mestrado em Zootecnia) - Universidade Federal de Santa Maria, 1999.

SIQUEIRA, E.R.; FERNANDES, S.; MESQUITA, V.S. et al. Efeito do peso ao abate sobre a eficiência de produção de cordeiros da raça Hampshire Down terminados em confinamento. In: REUNIÃO ANUAL DA SOCIEDADE BRASILEIRA DE ZOOTECNIA, 35., 1998, Botucatu. Anais... Botucatu: Sociedade Brasileira de Zootecnia, 1998. v.1. p.704-706.

SIQUEIRA, E.R. Sistemas de confinamento de ovinos para corte no Sudeste do Brasil. In: SIMPÓSIO INTERNACIONAL SOBRE CAPRINOS E OVINOS DE CORTE.1. 2000. João Pessoa. Anais... João Pessoa: 2000. p.107-118.

SILVA SOBRINHO, A.G.; BATISTA, A.M.; SIQUEIRA, E.R. et al. Nutrição de ovinos. Jaboticabal: FUNEP, 1996. 258p.

SUSIN, I.A.G.; BATISTA, A.M.; SIQUEIRA, E.R. et al. Nutrição de ovinos. Jaboticabal: FUNEP, 1996. 258p.

TOTHILl, J.C.; HARGREAVES, J.N.G.; JONES, R.M. BOTANAL: a comprehensive sampling and computing procedure for estimating pasture yield and composition. I. Field sampling. Tropical Agronomy Technical Memorandum, n.8, p.1-20, 1978.

ZUNDT, M.; MACEDO, F.A.M.; MARTINS, E.N. et al. Características de carcaça de cordeiros terminados em confinamento, alimentados com diferentes níveis proteicos. In: REUNIÃO ANUAL DA SOCIEDADE BRASILEIRA DE ZOOTECNIA, 38., 2001 Piracicaba. Anais... Piracicaba: Sociedade Brasileira de Zootecnia, 2001. p.988-990.

Recebido em: 28/06/02 Aceito em: 19/08/03

R. Bras. Zootec., v.33, n.1, p.225-233, 2004 